\title{
Advanced Networks in Motion Mobile Sensorweb
}

William D. Ivancic

Glenn Research Center, Cleveland, Ohio

David H. Stewart

Verizon Federal Systems, Cleveland, Ohio 


\section{NASA STI Program . . . in Profile}

Since its founding, NASA has been dedicated to the advancement of aeronautics and space science. The NASA Scientific and Technical Information (STI) program plays a key part in helping NASA maintain this important role.

The NASA STI Program operates under the auspices of the Agency Chief Information Officer. It collects, organizes, provides for archiving, and disseminates NASA's STI. The NASA STI program provides access to the NASA Aeronautics and Space Database and its public interface, the NASA Technical Reports Server, thus providing one of the largest collections of aeronautical and space science STI in the world. Results are published in both non-NASA channels and by NASA in the NASA STI Report Series, which includes the following report types:

- TECHNICAL PUBLICATION. Reports of completed research or a major significant phase of research that present the results of NASA programs and include extensive data or theoretical analysis. Includes compilations of significant scientific and technical data and information deemed to be of continuing reference value. NASA counterpart of peer-reviewed formal professional papers but has less stringent limitations on manuscript length and extent of graphic presentations.

- TECHNICAL MEMORANDUM. Scientific and technical findings that are preliminary or of specialized interest, e.g., quick release reports, working papers, and bibliographies that contain minimal annotation. Does not contain extensive analysis.

- CONTRACTOR REPORT. Scientific and technical findings by NASA-sponsored contractors and grantees.
- CONFERENCE PUBLICATION. Collected papers from scientific and technical conferences, symposia, seminars, or other meetings sponsored or cosponsored by NASA.

- SPECIAL PUBLICATION. Scientific, technical, or historical information from NASA programs, projects, and missions, often concerned with subjects having substantial public interest.

- TECHNICAL TRANSLATION. Englishlanguage translations of foreign scientific and technical material pertinent to NASA's mission.

Specialized services also include creating custom thesauri, building customized databases, organizing and publishing research results.

For more information about the NASA STI program, see the following:

- Access the NASA STI program home page at http://www.sti.nasa.gov

- E-mail your question via the Internet to help@ sti.nasa.gov

- Fax your question to the NASA STI Help Desk at $443-757-5803$

- Telephone the NASA STI Help Desk at 443-757-5802

- Write to: NASA Center for AeroSpace Information (CASI) 7115 Standard Drive Hanover, MD 21076-1320 


\section{Advanced Networks in Motion Mobile Sensorweb}

William D. Ivancic

Glenn Research Center, Cleveland, Ohio

David H. Stewart

Verizon Federal Systems, Cleveland, Ohio

Prepared for the

2011 Aerospace Conference

cosponsored by the Institute of Electrical and Electronics Engineers and the American Institute of Aeronautics and Astronautics

Big Sky, Montana, March 5-12, 2011

National Aeronautics and

Space Administration

Glenn Research Center

Cleveland, Ohio 44135 


\section{Acknowledgments}

The authors thank Cisco Systems for participating in a NASA Space Act Agreement to perform joint advanced protocol research; Mr. Pascal Thubert for his insight into the code; and Mr. Dan Shell for bringing the Duetto code to

NASA's attention and working on field demonstrations the early version of the Duetto code.

Contents were reproduced from the best available copy as provided by the authors.

Trade names and trademarks are used in this report for identification only. Their usage does not constitute an official endorsement, either expressed or implied, by the National Aeronautics and Space Administration.

Level of Review: This material has been technically reviewed by technical management.

Available from

NASA Center for Aerospace Information 7115 Standard Drive

Hanover, MD 21076-1320
National Technical Information Service 5301 Shawnee Road Alexandria, VA 22312

Available electronically at http://www.sti.nasa.gov 


\title{
Advanced Networks in Motion Mobile Sensorweb
}

\author{
William D. Ivancic \\ National Aeronautics and Space Administration \\ Glenn Research Center \\ Cleveland, Ohio 44135 \\ David H. Stewart \\ Verizon Federal Systems \\ Cleveland, Ohio 44135
}

\begin{abstract}
Advanced mobile networking technology applicable to mobile sensor platforms was developed, deployed and demonstrated. A two-tier sensorweb design was developed. The first tier utilized mobile network technology to provide mobility. The second tier, which sits above the first tier, utilizes 6LowPAN (Internet Protocol version 6 Low Power Wireless Personal Area Networks) sensors. The entire network was IPv6 enabled. Successful mobile sensorweb system field tests took place in late August and early September of 2009. The entire network utilized IPv6 and was monitored and controlled using a remote Web browser via IPv6 technology.

This paper describes the mobile networking and 6LowPAN sensorweb design, implementation, deployment and testing as well as wireless systems and network monitoring software developed to support testing and validation.
\end{abstract}

\subsection{Introduction}

The United States National Aeronautic and Space Administration (NASA) Earth Science Technology Office (ESTO) manages the development of advanced technologies and applications that are needed for cost-effective missions. ESTO plays a major role in shaping Earth science research and application programs of the future, aggressively pursuing promising scientific and engineering concepts, and ensuring that the program maintains an effective balance of investments in order to advance technology development. ESTO sponsored NASA's Glenn Research Center (GRC) to research and deploy advanced mobile networking technology applicable to mobile sensor platforms. As a result, GRC personnel developed a two-tier sensorweb design. The first tier utilized mobile ad hoc network (MANET) technology to provide mobility. The second tier, which sits above the first tier, utilized 6LowPAN (Internet Protocol version 6 Low Power Wireless Personal Area Networks) sensors. The entire network was IPv6 enabled.

This paper describes the mobile networking and 6LowPAN sensorweb design, implementation, deployment and testing as well as wireless systems and network monitoring software developed to support test and validation. In addition, issue in deployment and idiosyncrasies of various technologies and tools are identified and discussed.

\subsection{Mobile Networking}

GRC collaborated with Cisco System, Incorporated to research and deploy advanced mobile networking technology applicable to mobile sensor platforms. GRC personnel developed a two-tier sensorweb design. The first tier utilized mobile network technology from Cisco Systems to provide mobility. GRC utilized a combination of mobile IP and mobile ad hoc networking alpha software from Cisco System, known internally to Cisco as "Duetto". ${ }^{1}$ Duetto covered implementation of the mobile router functionality for Mobile IPv6 and nested NEMO (NEtworks in MOtion) support. In addition, Duetto performed full Tree Discovery and MANET (Mobile Ad hoc NETwork) support called "Bubbles". Portions of the "Bubbles" protocol has applications in low power sensorwebs for smart buildings, industrial controls and monitoring and has been incorporated into specifications being developed by the Internet Engineering Task Force working group on Routing Over Low power and Lossy networks (roll) (Ref. 1).

Some of the major features of this software build include: tree discovery, nemo support, and Reverse Routing Header (RRH). Tree Discovery establishes a tree using extended IPv6 Neighbor Discovery. Neighbor Discovery occurs at the speed of the link layer (L2); therefore, the tree discovery occurs very quickly. The nemo portion of the Duetto code exploits the tree to optimally get out of a nested set of Mobile Routers (MRs) and register to the mobile-ip Home Agent. RRH extends the nemo support to provide route optimization and added security. The "Bubbles" or MANET portion of the Duetto code also extends Neighbor discovery in order to quickly establish the routes down the cluster. Finally, since "Bubbles" and the nested mobile networks (nested nemo) both exploit the same cluster (tree), switching back and forth from Mobile IP (nemo) to ad-hoc "Bubbles" is optimized.

The Duetto software was originally debugged by Cisco Systems personnel using a wired network. GRC personnel implemented a wireless network with radios on each interface.

\footnotetext{
${ }^{1}$ This code was developed by Cisco System France. Primary designers include Pascal Thubert and Marco Molteni. The code was Cisco IOS Software, 3200 Software (C3250-ADVENTERPRISEK9M), Experimental Version 12.4(20060331:114112) [pthubert-clairette 168]
} 
Numerous problems were uncovered in the wireless network that did not show up in the wired network. These problems were rectified by either code fixes performed by Cisco, network configurations or readdressing the networkwhichever was the appropriate corrective action.

The Duetto code was installed on Cisco 3200 series Mobile Access Routers Cards (MAR), which utilize the PC/104-Plus ${ }^{2}$ hardware standard. In addition to the router, a Fast Ethernet Mobile Switched Interface Card (FESMIC) was used as were two Cisco C3201 Wireless Mobile Interface Cards (WMIC). ${ }^{3}$ A WMIC is connected to the ingress interface (FESMIC, vlan1) and its station-role set to "root bridge" mode making this a "Parent" radio. A separate WMIC radio was connected to the egress or wan interface $(\mathrm{Fa}-0 / 0)$ and station-role was set to "workgroup-bridge" making this a "Child" radio. The "root bridge"/"working group" pair was the only mode found to work with IPv6. ${ }^{4}$ Other Combinations broke the IPv6 stateless auto-configuration, which is necessary for the Duetto protocol. In addition the switch ports (FESMIC ports) on the MAR had to have spanning-tree PortFast set. Spanning-tree PortFast causes a port to enter the spanning-tree forwarding state immediately, bypassing the listening and learning states.

Figure 1 shows a typical mobile node. The egress interface is connected to a wireless radio configured in "Child" mode. This radio can connect to a single "Parent" radio to form a tree structure. Likewise, the Ingress interface is connected to a radio configured to be a "Parent". Note: many mobile nodes can connect to a single "Parent" as a single "Parent" can simultaneously associate with multiple Children.

In a wired network, the ingress and egress inputs never connect to each other and thus never see each other. In a wireless system, the MANET radio, ${ }^{5}$ which resides on the egress port, can see a MANET radio attached to its own ingress port. Cisco implemented a loop avoidance algorithm in the Tree Discovery process to ensure that the mobile networking software prohibited a single node's egress port(s) from connecting to its ingress port as well as ensure that a Top Level Mobile Router (TLMR) ${ }^{6}$ did not connect to a node that is deeper in its own tree. Even so, we still encountered problems with layer-2 radio loops. Each mobile network pair had to be configured to ensure that the "Child" radio was

\footnotetext{
${ }^{2}$ Due to the combined power requirements exceeding that of the power supply (Datel MAPC-104) and the PC/104-Plus standards, the WMICs were in a separated PC-104 stack.

${ }^{3}$ The Cisco 3201 WMIC is a $2.4 \mathrm{GHz}, 802.11 \mathrm{~b}$.g radio.

${ }^{4}$ WMIC cards are not capable of full MANET radio functionality. Bridge-Mode was the only combination found to work. An exhausted amount of testing of various configurations was performed. However, there were several issues that were resolved during testing (i.e. spanning tree, RFI, etc.). There may be other configurations that will work with IPv6, however, this is one that configuration that definitely will work:

${ }^{5} \mathrm{~A}$ true MANET radio will listen and communicate with all other MANET radios.

${ }^{6}$ TLMR refers to the root router of the topology tree that is formed. For example, router 509 in figure 8 is the TLMR of that tree.
}

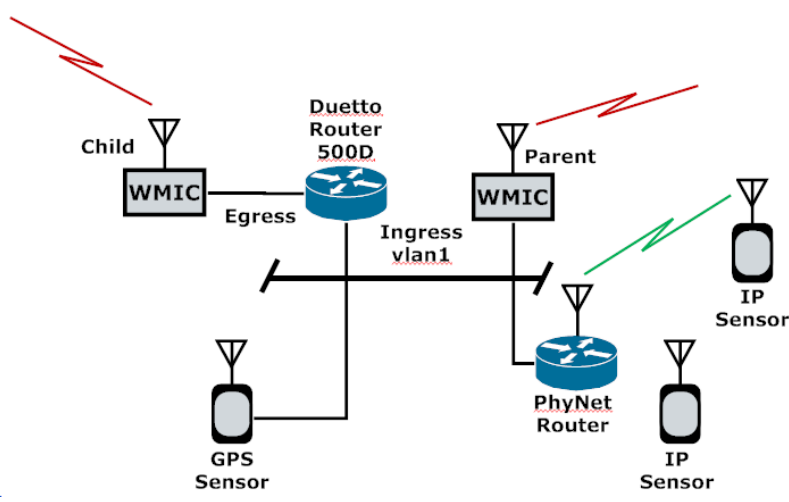

Figure 1.-IPv6 mobile node with radio.

prohibited from associating with the "Parent" radio on the same mobile node. It is possible for two mobile routers to become isolated because the "Parent" radio of router A attaches to the "Child" radio of router B and the "Parent" radio of router B attaches to the "Child" radio of router A. ${ }^{7}$ This is a legitimate configuration - particularly if only two systems exist. The Duetto code will make one of those two nodes the TLMR. However, those two systems become isolated from the rest of the group. In order to solve this problem, one would have to have some interaction between the radio system and the router (layer-2/layer3 interaction) such that if the router realized that a single layer loop has occurred, then, the layer-3 routing software would notify the radio whereupon that radio would then to try and reassociate with a different radio.

For demonstration purposes, we could configure the radios to prevent specific parent/child pairs from associating with each other to avoid routing loops caused by layer-2 radio associations. This helped demonstrate the layer-3 mobile networking Duetto code, but is not a scalable solution. The WMICs also appeared to have a feature whereby one could prioritize the Service Set Identifications (SSIDs). However, we could not get that feature to work in both "Parent" and "Child" radios. Furthermore, even if this would have enabled us to prevent some isolation loops, it is also not a scalable solution and requires a priori knowledge of the network and possible contacts between nodes.

Another problem that was uncovered was that the initial configurations showed proper route tables in the MAR routers and that all routes appearing to be accessible. However, during testing, it was apparent that not all nodes were truly reachable even though the route tables indicated otherwise. Troubleshooting the system uncovered the following:

1. The radios were turned on and "Child" nodes would associate to a parent.

2. Initially the egress (fa0/0) interface would get a IPv6 address from a Routing Advertisement (RA) with a default lifetime of $1800 \mathrm{sec}$.

${ }^{7}$ This scenario did not occur often when the MANET nodes were setup in the lab, but is was a common occurrence during the migration to the outdoors deployment. 
3. Spanning tree would kick in and block the connection.

4. Bubbles would think it had a valid address and maintain an entry in the routing table, even though the connection was blocked.

5. 30 min later the RA would expire, spanning tree was still blocking so the entry would be deleted from the routing table.

The solution was to do the following in the router configurations:

1. RA lifetime was reduced to $10 \mathrm{sec}$

2. Spanning-tree portfast was enabled on the switching interfaces of the routers.

3. Layer-2 spanning-tree was disabled in the WMICs. (Although not documented as a fix to this problem, the command is in the WMIC's configuration file.)

An interesting feature of the Duetto code is that the network and tree discovery are performed using IPv6 features. Once a tree is established using IPv6, the code propagates IPv4 routes. The code forwards its routing table out its egress interface to the next highest-level router in the tree (this includes the bubbles entries along with any subnets that are defined on its interfaces). The code also passes the IPv4 routing table thereby creating an IPv4 MANET in addition to the IPv6 MANET. This was discovered by accident, but proved extremely useful. The Cisco PC 104 Wi-Fi cards, WMICs, are not IPv6 capable so IPv4 address were assigned for administrative purposes. One could then access a router via IPv6 and then telnet from the router to the locally attached WMIC via IPv4. It was discovered after the bubbles network was functional that the WMICs could be accessed from any node on the system using IPv4 addressing.

\subsection{Routing Nuances}

The "Bubbles" portion of the Duetto code uses auto configuration to create the tree structure. Each egress interface is set to auto configure and is connected to a radio in "child" mode. Each ingress interface has a fixed /64 address and sends a route advertisement out. Each ingress interface is also connected to a "Parent" radio. When a "Child" radio attaches to a "Parent" radio it receives a new RA and becomes part of the Parent's network. The IPv6 auto configuration on the IOS version used would only work if the subnet of the advertising interface was a /64. Therefore each egress IPv6 interface needed to be configured with a /64 subnet.

It is important to note that the Duetto code only forwards subnets assigned to its interfaces and any subnet entries received from routers farther down the tree. The Duetto code does not propagate static routes (static route redistribution) or subnets assigned to tunnels.

\subsection{Routing to the 6LowPAN Sensors}

The 6LowPAN network is described in the next section, Section 3.0, IPv6 Sensorweb. However, the routing is described here.

The 6LowPAN network consists of a server, 6LowPAN routers and 6LowPAN sensors. The routers and server were attached to Ethernet interfaces on the mobile ad hoc routers, the Cisco MAR routers (Fig. 1). In the case of the 6LowPAN router, it has two interfaces, an egress and ingress interface. The egress interface of the 6LowPAN router is connected to the ingress interface of the ad hoc router subnetwork. The ingress interface of the 6LowPAN router is connected to the 6LowPAN sensors - often via the 802.15.4 wireless link. The ad hoc router must provide address space to accommodate at least two subnetworks: one for the 6LowPAN egress interface and one for the 6LowPAN ingress interface. In order to accomplish this, a secondary address is implemented on the ad hoc router ingress interface providing a /63 subnet for the 6LowPAN router and sensors. This subnet "effectively", not literally, is split into two /64 subnets with one /64 subnet allocated to the 6LowPAN sensors. However, in order for the ad hoc router to reach the /64 subnet of the 6LowPAN network, a static route is also needed. The following is from an actual configuration:

interface Vlan1

description "INGRESS or ATTACHMENT INTERFACE"

ip address 10.50.13.1 255.255.255.0

ipv6 address FDAD:9F5B:4B7D:500D::1/64

ipv6 address FDAD:9F5B:4B7D:50D0::1/63

ipv6 route FDAD:9F5B:4B7D:50D1::/64

FDAD:9F5B:4B7D:50D0::77

Note: the 10.50.13.0/24 IPv4 subnet actually gets propagated up the tree and will show up in the routing table.

- FDAD:9F5B:4B7D:500D::/64 is the subnet that other ad hoc routers attach to via their "Child" radio. This is the subnet that is advertised using router advertisements.

- FDAD:9F5B:4B7D:50D0::/63 is an additional subnetwork that is propagated up the tree. The secondary interface address on the ad hoc router ingress interface is FDAD:9F5B:4B7D:50D0::1. For consistency, the egress interface of the 6LowPAN router was always given an address in the lower portion of the /63 subnet with the last 64 bits as 77 . In this example, the 6LowPAN router's egress interface is FDAD:9F5B:4B7D:50D0::77. The 6LowPAN router is configure to have its ingress interface use the upper half of the /63 subnet or FDAD:9F5B:4B7D:50D1::/64. The route statement in the configuration enables the ad hoc router to understand where to send data destined to the 6LowPAN sensors. ${ }^{8}$

${ }^{8} \mathrm{~A}$ static address has to be used for the following reasons: Since the IPv6 subnet was a /63, the router would not support auto-configuration. The address had to be known so an IPv6 static route could be preconfigured. Also, the 6LowPAN router was administered via HTTP so it had to be at a known address. 


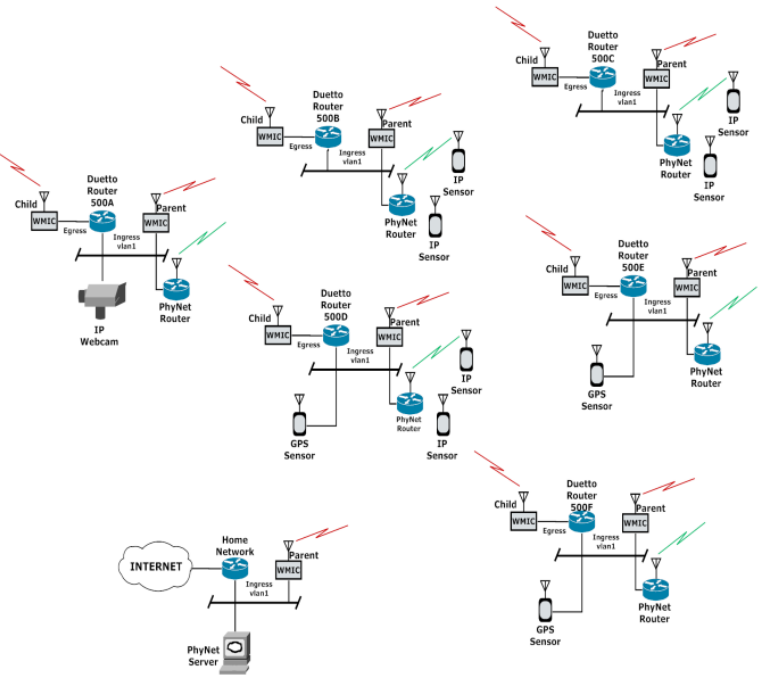

Figure 2.- “Bubbles" IPv6 mobile ad hoc network.

The seven node IPv6 mobile ad hoc network is shown in Figure 2. One node is connected to the Internet to provide connectivity to the general Internet. This connectivity was via and IPv4 network while the mobile IPv6 mobile ad hoc network was configured to use IPv6 Unique Local Addressing to ensure that no experimental traffic would leak to the open Internet. Each mobile node had one "Parent" radio and one "Child" radio. Thus any mobile node could become the toplevel mobile router (TLMR) depending on the movement and the RF connectivity of the devices. For our test network, the two extreme limits for forming tree structures are: 1) a single serial string of 7 routers (i.e., 6 layers deep); or 2) one toplevel router with 6 children (i.e., 1 layer deep).

\subsection{IPv6 Sensorweb}

In order to demonstrate a mobile sensorweb, a search was performed to identify potential IPv6 sensors. Numerous 6LowPAN sensors were identified. ArchRock IPv6 sensors (Ref. 2) were procured and integrated into the mobile ad hoc network. These sensors were configured to be within the address space of the mobile ad hoc network forming a second tier network sitting above the first tier. The entire nemo mobile IP network and "Bubbles" ad hoc network along with the sensor network were all connective via IPv6 addressing.

The 6LowPAN sensor network that was constructed consisted of a server, 3 6LowPAN routers and numerous 6LowPAN sensors. The server manages interconnected collections of IP-based wireless sensor networks using a common web services architecture and web browser interface. The server was located in the Protocol Research Lab (bldg. 54) and connected to the 6LowPAN routers via the Cisco IPv6 MANET network. The routers are IP-based 802.15.4 wireless sensor networking devices that connect 6 LowPAN mesh networks to the main server via Wi-Fi and Ethernet interfaces. In our setup, the 802.15.4 wireless links

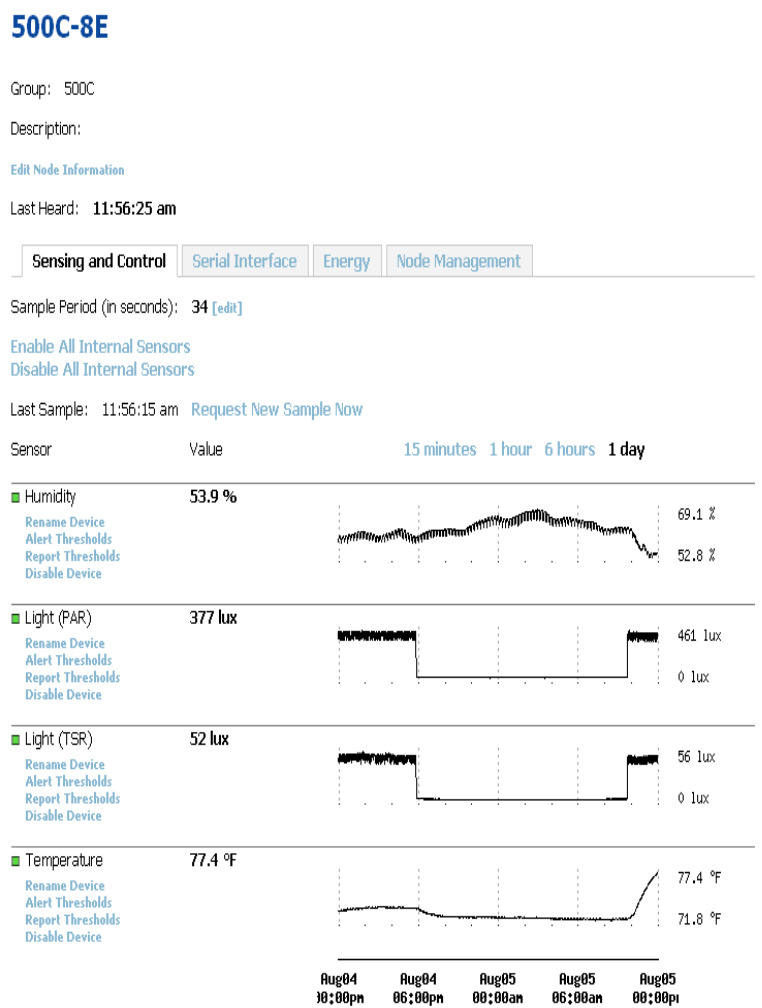

Figure 3.-Wireless personal area networks sensor (time scale $24 \mathrm{hr}$ from midnight to midnight in $6 \mathrm{hr}$ increments).

connected the 6LowPAN sensors to the router and an Ethernet interface was connected to the "Bubbles" MANET router. The ArchRock Wi-Fi link was not used.

The ArchRock server keeps track of all 6LowPAN routers and nodes and maintains status and history of the sensors, with the exception of the ArchRock IP serial Nodes. A Linux server that also provided the topology drawings queried the IP serial nodes. This Linux server was co-located with the 6LowPAN server.

The ArchRock server standard configuration uses virtual private networks (VPNs) to connect to the 6LowPAN routers. There was a bug in the ArchRock server code relative to the use of VPNs over IPv6. In order to keep everything in the network on IPv6 address space, VPNs were abandoned (IPv4 addresses were configured, but only for debugging the VPN). The routing configuration for this has previously been described in Section 2.0, Mobile Networking.

Sensors for temperature, power, humidity and luminance were used in this testbed for demonstration purposes. These sensors also monitored the voltage of the battery pack that powered the transportable MANET nodes. These 6LowPAN sensors were selected because they provided some data that could easily be correlated to the conditions at hand.

One sensor group was located in a cargo van that we could drive throughout Glenn Research Center. Figure 3 shows the detail readout of that particular sensor. The sensor shown is 


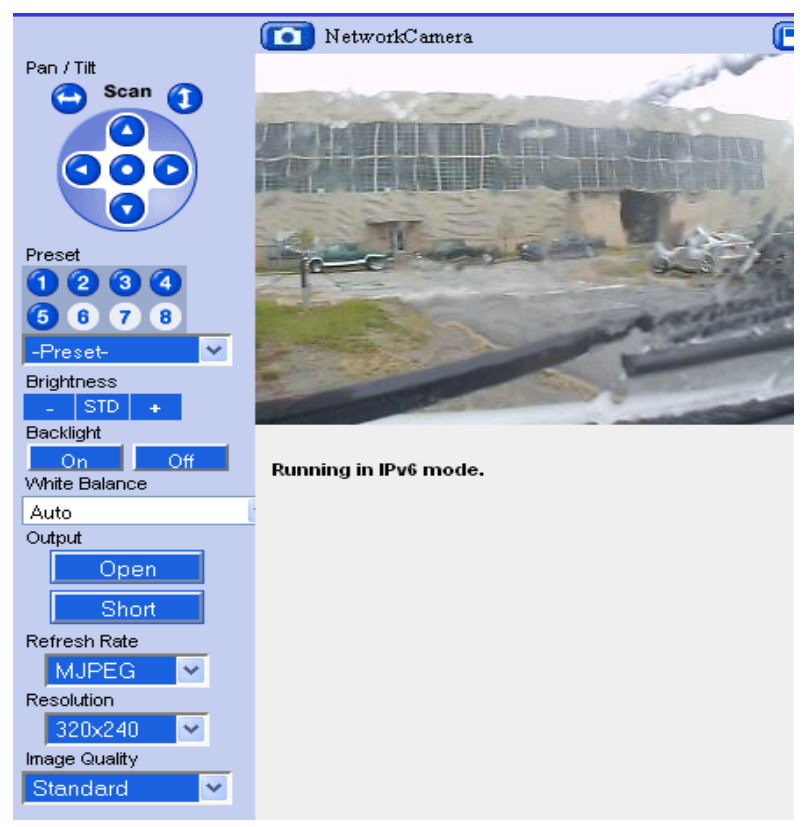

Figure 4.-Panasonic IPv6 WebCAM (rainy day at Glenn Research Center).

500C-8E, which was located inside the cargo compartment of the mobile van. From the sensor readings one can determine that the lights were off from 6:00 p.m. to about 9:00 a.m. (second reading from the bottom) and the temperature increased soon after the Sun came up at approximately 9:00 a.m. (bottom reading).

In addition to the 6LowPAN sensors, an IPv6 enabled Webcam was installed on the van. A Panasonic Webcam was used. One undocumented nuance with this particular device was that the camera had to be configured with an IPv4 address first, then one could configure the IPv6 address. Furthermore, this had to be done in the first 10 to 20 min of startup or the camera locked out changes of its configuration, for security reasons.

Figure 4 shows the output of the IPv6 Webcam attached to Mobile Node 500C, the mobile VAN. This Webcam was controlled using IPv6.

\subsection{Field Tests}

The mobile sensorweb system field tests took place in late August and early September of 2009. Figure 5 shows a conceptual field deployment. Here, we only needed to have one mobile unit in order to make the entire network topology change. Hence "network mobility" was exercised at each mobile router due to changes in network topology as the van moved about the GRC campus. In order to remotely monitor each mobile node, we configured the network such that at certain points in the movement of van, every node was reachable. This was not necessary for the network to function. Rather, this was done as a matter of convenience for testing and debugging.

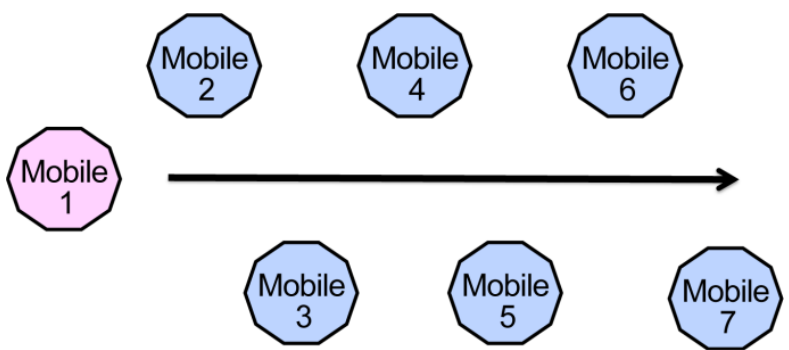

Figure 5.-Mobile network notional test deployment.

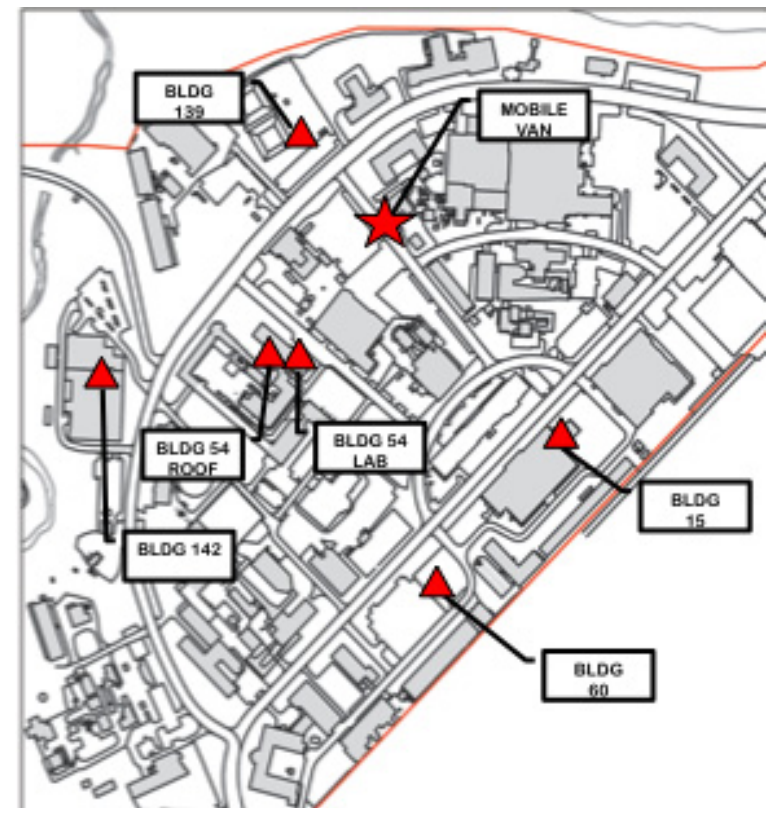

Figure 6.-MANET router locations.

Figure 6 shows the locations of the seven MANET router nodes place throughout NASA's Glenn Research Center. The "Star" indicates the van. As the van moves around the lab, the wireless connectivity changes and the MANET topology changes. Two MANET routers were co-located in the Protocol Lab (bldg. 54). One of these routers (router 5009) had external antennas mounted on the roof of the building in order to connect with the other wireless systems scattered throughout the GRC campus. It is important to note, not all nodes could communicate directly with each other. In order to connect nodes that did not have direct RF connectivity, these nodes had to hop through others. This was done intentionally to exercise the full capabilities of the Duetto code.

In order to monitor the mobile nodes, GRC developed a simple network management system, which showed location of the nodes and simple status. That system consisted of two independent pieces: a location system, which used GPS (Global Positioning System); and, a network monitoring system, which was implemented using PERL (Practical Extraction and Report Language). 


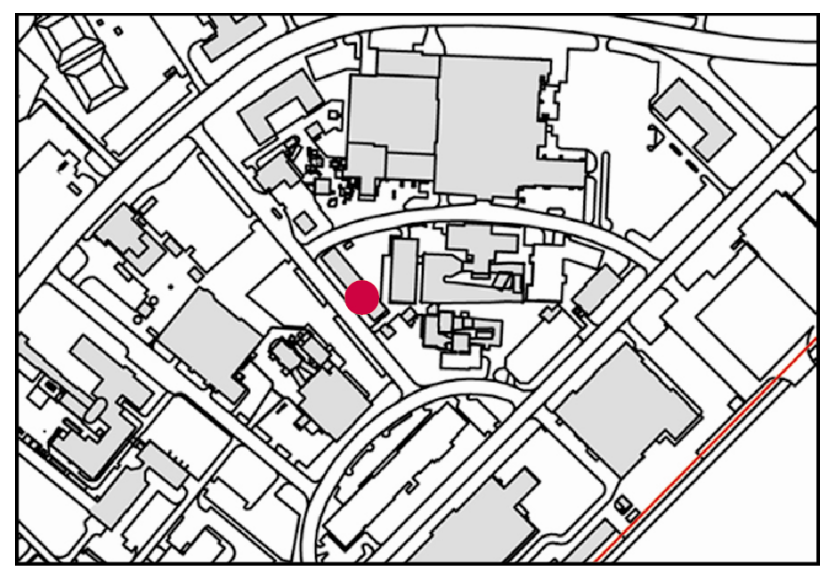

Figure 7.-GPS location of 500C Van Node (IPv6).

The first piece utilized GPS and the 6LowPAN sensorweb serial devices to obtain GPS data over the network. A Linux computer polled the GPS device and utilizes Google Maps to map the location of the GPS receiver onto a map of Glenn Research Center. The GPS receiver was placed in the van to show the location of the van as it travels around the GRC campus. This provided a reasonable visual check to see if network topology changes match what would be expected from the RF link possibilities at any given instant in time. In Figure 7 , the location of the van, mobile node $500 \mathrm{C}$, is depicted by the red dot in the middle of the map.

\subsection{Tracking and Monitoring Tool}

A PERL based network monitoring tool was developed and used to display the network topology of the 7-node network. The PERL script used Graphvis (Ref. 3) to display the network topology. Figures 8 and 9 show two configurations of the same seven-node network along with the 6LowPAN sensors. The 6LowPAN routers (light blue boxes) are labeled "PhyNet Router" and the 6LowPAN sensors are labeled "IP sensor". The IPv6 mobile ad hoc (MANET) routers use Unique Local Address (ULA) to ensure testbed routes do not propagate to the Internet. That address space is FDAD:9F5B:4B7D::/48. We further subnet this space down to /64 addresses for the mobile ad hoc network routers and /63 subnetworks for each 6LowPAN router and its associated sensors - see "Routing to the 6LowPAN Sensors" in Section 2.0, Mobile Networking. The routers are labeled according to the last 16 bits of their /64 subnet. For example: 500A Router is the router on subnet FDAD:9F5B:4B7D:500A. By our own convention, the $/ 63$ subnet is associated with an ad hoc router that has a 6LowPAN network attached is FDAD:9F5B:4B7D:50X0/63 where " $\mathrm{X}$ " is a alpha character associated with the ad hoc ingress /64 subnet. All Vlan1 interface addresses are: 50X0::1 and all PhyNet router addresses are: 50X0::77. The Webcam is located in the van node on subnet: $500 \mathrm{C}:: / 64$.

The node labeled "5009" is located in our protocol lab (bldg. 54) and physically never moves. It is attached to the open Internet. The 6LowPAN server is attached to this router. The router labeled " $500 \mathrm{C}$ " is the van. As the van moves about the GRC campus, the network topology changes, often leaving some nodes isolated. Note: changes in network topology can occur due to movement of nodes or simply changes in RF link characteristics due to blockage, atmospheric conditions, interference, or other factors.

Figure 8 shows a mobile ad hoc network with a tree depth of three. Note that the mobile router " $500 \mathrm{E}$ " at building 142 is isolated. No other systems are in contact with this route as it was intentionally positioned to only become connected to the entire network if the mobile node (Van) came close enough to close the RF links. Also, in Figure 8, the top-level router is the router located in building 54 and connected directly to the Internet.

Figure 9 shows a change in topology from Figure 8 . The ad hoc router at building 142 is now connected to the rest of the network via the ad hoc router in the van. The tree depth here is 4 and the top-level ad hoc router is the building 54 lab router, which has a 6LowPAN router attached. This 6LowPAN router has two 6LowPAN sensors attached to it. Only mobile ad hoc routers with 6LowPAN servers or routers required additional network address space- - see Section 2.0 subsection "Routing to the 6LowPAN Sensors".

\subsection{Sensorweb Discovery}

Web services and sensor discovery were not implemented in this demonstration. However, since one MANET router, the router located in the Protocol Lab, was connected to the Internet and since the ArchRock server was also tied to this MANET router, the entire system was reachable via the Web. Furthermore, since this router node was always connected to the same Internet address, we would have easily registered that address in the Internet Domain Name System (DNS). Thus, one should be able to access the mobile sensorweb information using the Open Geospatial Consortium (OGC) specifications and standards (Ref. 4) so long as security accesses privileges were granted. 


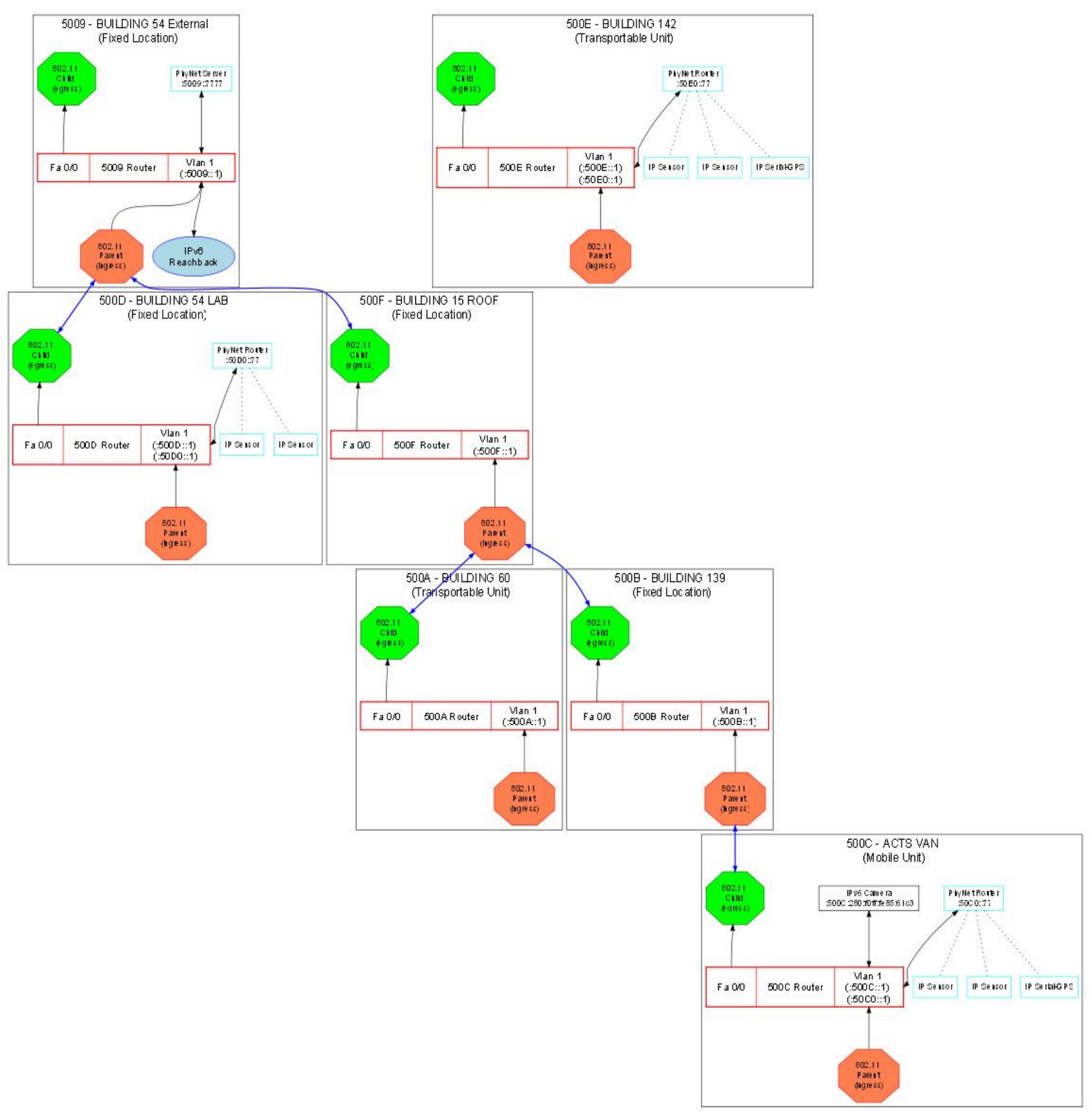

Figure 8.-Mobile network topology 1. 


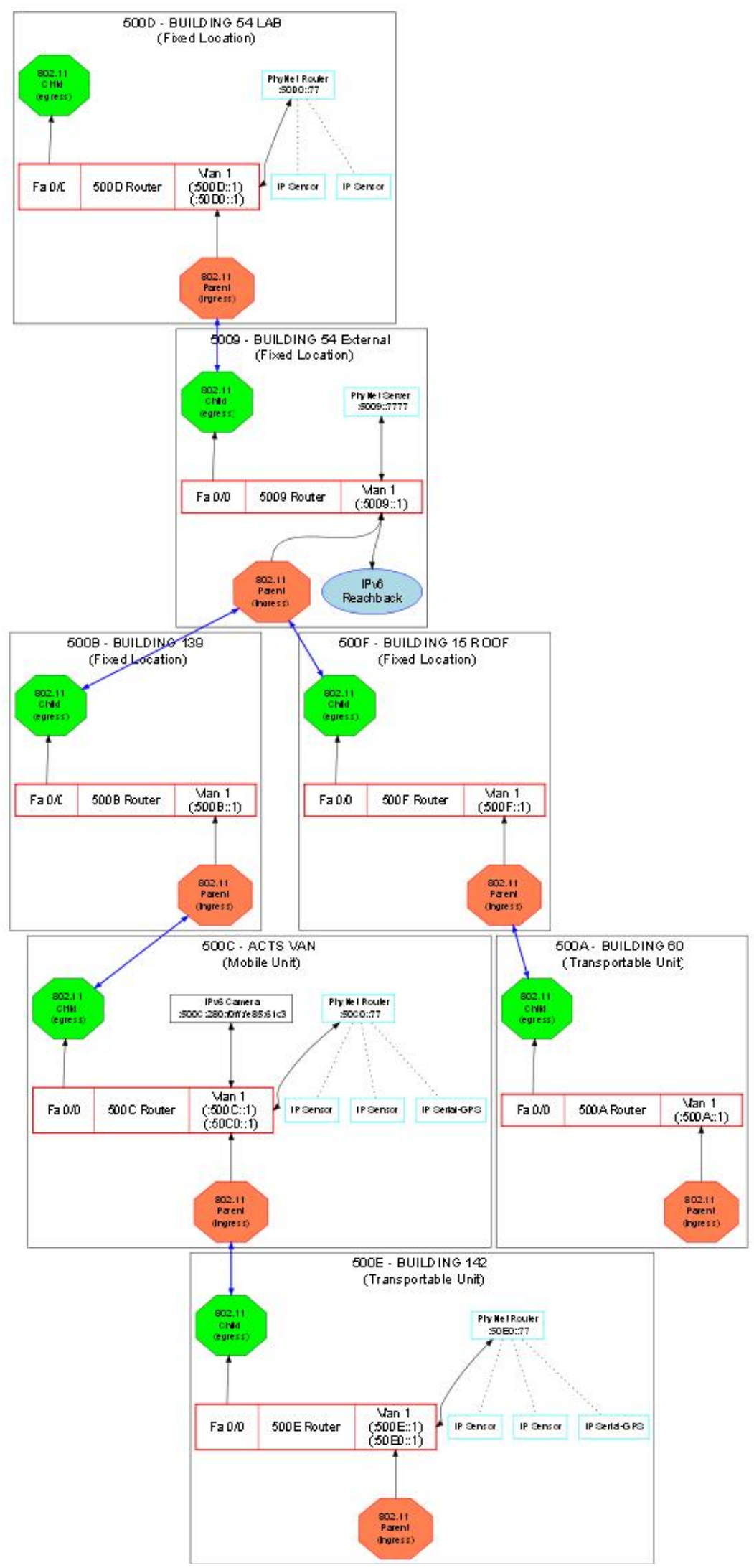

Figure 9.-Mobile network topology 2. 


\subsection{Conclusions}

A two-tier sensor network was successfully demonstrated that utilized an ad hoc mobile network to handle mobility and a 6LowPAN sensor network to provide sensor readings. The entire system operated using IPv6 technology. In addition, and IPv4 mobile network could be constructed but only if IPv6 was operational as IPv6 neighbor discovery is used to discover and construct the ad hoc network.

A parent/child system was used in this network. Such a system could result in mobile ad hoc router pairs becoming isolated. Therefore, some interaction between the layer-3 mobile ad hoc networking code and the radio system should be considered to help alleviate this problem.

There continues to be a need for a radio that is developed specifically to work with layer-3 ad hoc and mobile networking rather than in conflict.

\section{References}

1. T. Winter, P. Thubert, "RPL: IPv6 Routing Protocol for Low power and Lossy Networks." IETF draft-ietf-rollrpl-11 work in progress July 28, 2010

2. http://www.archrock.com/, October 2010

3. http://www.graphviz.org/, October 2010

4. http://www.opengeospatial.org/, October 2010

\section{Biography}

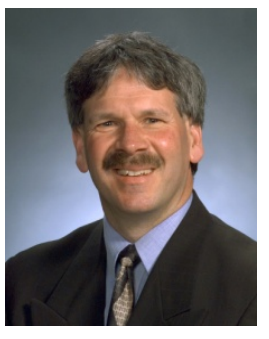

William Ivancic has over twenty-seven years of experience in network and system engineering for communication applications, communication networking research, state-of-the-art digital, analog and RF hardware design and testing. He currently is a senior research engineer at NASA's Glenn Research. Of particular interest is large scale, secure deployment of mobile networks including mobile-ip and mobile router technology. Mr. Ivancic is currently working on communication protocols and network designs to improve the data delivery for Unmanned Aeronautic Vehicles (UAVs).

Mr. Ivancic is also principle of Syzygy Engineering, a small consulting company specializing in communications systems and networking as well as advanced technology risk assessment. Mr. Ivancic is currently performing research and development on Identity-based security and key and policy management and distribution for tactical networksparticularly mobile networks.

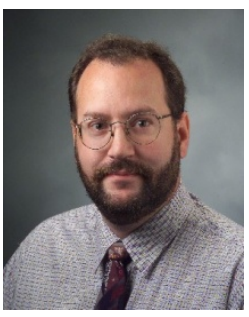

David Stewart is a communication engineer at Verizon. Mr. Stewart specializes in RF and wireless communication networks. His current work involves development and deployment of secure mobile networking technologies in various testbeds at NASA's Glenn Research Center, as well as deployment of early-field-trial aeronautic and space-based networks. In addition, Mr. Stewart is developing and testing reliable transport protocols for large file transfers as well as store-and-forward technologies. 


\begin{tabular}{|c|c|c|}
\hline \multicolumn{2}{|c|}{ REPORT DOCUMENTATION PAGE } & $\begin{array}{c}\text { Form Approved } \\
\text { OMB No. 0704-0188 }\end{array}$ \\
\hline \multicolumn{3}{|c|}{ 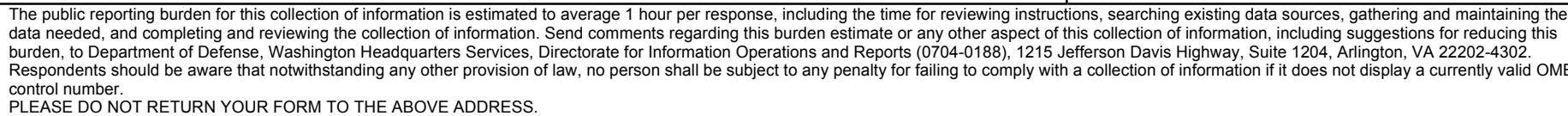 } \\
\hline $\begin{array}{l}\text { 1. REPORT DATE (DD-MM-YYYY) } \\
01-03-2011\end{array}$ & $\begin{array}{l}\text { 2. REPORT TYPE } \\
\text { Technical Memorandum }\end{array}$ & 3. DATES COVERED (From - To) \\
\hline \multirow{3}{*}{\multicolumn{2}{|c|}{$\begin{array}{l}\text { 4. TITLE AND SUBTITLE } \\
\text { Advanced Networks in Motion Mobile Sensorweb }\end{array}$}} & 5a. CONTRACT NUMBER \\
\hline & & 5b. GRANT NUMBER \\
\hline & & 5c. PROGRAM ELEMENT NUMBER \\
\hline \multirow{3}{*}{\multicolumn{2}{|c|}{$\begin{array}{l}\text { 6. AUTHOR(S) } \\
\text { Ivancic, William, D.; Stewart, David, H. }\end{array}$}} & 5d. PROJECT NUMBER \\
\hline & & 5e. TASK NUMBER \\
\hline & & $\begin{array}{l}\text { 5f. WORK UNIT NUMBER } \\
\text { WBS 430728.02.05.05.01 }\end{array}$ \\
\hline \multicolumn{2}{|c|}{$\begin{array}{l}\text { 7. PERFORMING ORGANIZATION NAME(S) AND ADDRESS(ES) } \\
\text { National Aeronautics and Space Administration } \\
\text { John H. Glenn Research Center at Lewis Field } \\
\text { Cleveland, Ohio 44135-3191 }\end{array}$} & $\begin{array}{l}\text { 8. PERFORMING ORGANIZATION } \\
\text { REPORT NUMBER } \\
\text { E-17629 }\end{array}$ \\
\hline \multirow{2}{*}{\multicolumn{2}{|c|}{$\begin{array}{l}\text { 9. SPONSORING/MONITORING AGENCY NAME(S) AND ADDRESS(ES) } \\
\text { National Aeronautics and Space Administration } \\
\text { Washington, DC 20546-0001 }\end{array}$}} & $\begin{array}{l}\text { 10. SPONSORING/MONITOR'S } \\
\text { ACRONYM(S) } \\
\text { NASA }\end{array}$ \\
\hline & & $\begin{array}{l}\text { 11. SPONSORING/MONITORING } \\
\text { REPORT NUMBER } \\
\text { NASA/TM-2011-216990 }\end{array}$ \\
\hline \multicolumn{3}{|c|}{$\begin{array}{l}\text { 12. DISTRIBUTION/AVAILABILITY STATEMENT } \\
\text { Unclassified-Unlimited } \\
\text { Subject Category: } 04 \\
\text { Available electronically at http://www.sti.nasa.gov } \\
\text { This publication is available from the NASA Center for AeroSpace Information, 443-757-5802 }\end{array}$} \\
\hline
\end{tabular}

\section{SUPPLEMENTARY NOTES}

\section{ABSTRACT}

Advanced mobile networking technology applicable to mobile sensor platforms was developed, deployed and demonstrated. A two-tier sensorweb design was developed. The first tier utilized mobile network technology to provide mobility. The second tier, which sits above the first tier, utilizes 6LowPAN (Internet Protocol version 6 Low Power Wireless Personal Area Networks) sensors. The entire network was IPv6 enabled. Successful mobile sensorweb system field tests took place in late August and early September of 2009. The entire network utilized IPv6 and was monitored and controlled using a remote Web browser via IPv6 technology. This paper describes the mobile networking and 6LowPAN sensorweb design, implementation, deployment and testing as well as wireless systems and network monitoring software developed to support testing and validation.

\section{SUBJECT TERMS}

Communication; Networking

\begin{tabular}{|c|c|c|c|c|}
\hline \multicolumn{3}{|c|}{ 16. SECURITY CLASSIFICATION OF: } & \multirow{2}{*}{$\begin{array}{l}\text { 17. LIMITATION OF } \\
\text { ABSTRACT } \\
\text { UU }\end{array}$} & \multirow{2}{*}{$\begin{array}{l}\text { 18. NUMBER } \\
\text { OF } \\
\text { PAGES } \\
15\end{array}$} \\
\hline $\begin{array}{l}\text { a. REPORT } \\
U\end{array}$ & $\begin{array}{l}\text { b. ABSTRACT } \\
U\end{array}$ & $\begin{array}{l}\text { c. THIS } \\
\text { PAGE } \\
\text { U }\end{array}$ & & \\
\hline
\end{tabular}



